\title{
New Solution Implementation to Protect Encryption Keys Inside the Database Management System
}

\author{
Karim El bouchti*, Soumia Ziti, Fouzia Omary, Nassim Kharmoum \\ Faculty of Sciences, Mohammed V University in Rabat, Intelligent Processing Systems \& Security (IPSS) Team, Morocco
}

\begin{tabular}{l} 
A R T I C L E I N F O \\
\hline Article history: \\
Received: 31 December, 2019 \\
Accepted: 13 February, 2020 \\
Online: 09 March, 2020 \\
\hline
\end{tabular}

Keywords:

Encryption keys protection

Database encryption

Database security

keys protection in Databases

\begin{abstract}
A B S T R A C T
Due to the attacks' growth on sensitive databases by deploying advanced tools, beyond access control and authentication mechanisms, the database encryption remains a useful and effective way to ensure robust security of data stored within it. Any database encryption solution is based on a specific encryption model that determines how data is encrypted inside it. A relevant database encryption model must necessarily adopt a strong security policy of data encryption keys. It determines how these keys are generated, stored, and protected. In this work, we will implement an original solution that protects the encryption keys when encrypting data occurred at the Database Management System level. Our solution suggests protecting the keys by their encryption with other ones named Master Keys, which are generated according to the encryption granularity defined by the database encryption model. The proposed solution protects the keys of two database encryption models: at the level of the columns and the level of tables.
\end{abstract}

\section{Introduction}

Database (DB) level encryption is a way to encrypt and decrypt data within the Database Management System (DBMS) using keys held by the DB server [1]. In fact, this encryption model offers major advantages, in particular, those related to the security of the encryption keys against external attacks performed outside the information system [2, 3]. Though the probability of the administrator attack is not negligible, he owns large privileges on DB. Hence, he can attack the DB directly, via a collaboration with a malicious external attacker (disclosure of the administrator account for example), or with an internal attacker such as a legitimate user $[4,5,6]$. Obviously, the DB administrator has the ability to perform all these attacks without leaving any traces [7, 8].

Data encryption at the DBMS level is based fundamentally on its specific encryption model. The management of encryption keys within this model is a crucial point, it defines the ways how keys are generated, stored, and protected $[9,10]$. Actually, keys values, how users access them, and where they are stored are the ultimate goal of any attacker. Therefore, it should be necessary to establish a protection policy for encryption keys to minimizing their exposure face of malicious attackers. Indeed, many solutions have

${ }^{*}$ Karim El bouchti, Email: elbouchtikarim@gmail.com been developed in order to resolve this problem. For instance, the keys protection solutions implemented at DBMSs such as Oracle, Ms SQL Server and My SQL are mainly based on the use of "Wallets", Hardware Security Module (HSM) and "Security server" [11-14]. In fact, each one of these solutions has advantages and major limits, as have already explained in more detail in our previous work $[8,15]$.

Several studies proposed several solutions to protect DB encryption keys either in DB encryption models or separate solutions of keys protection [16, 17]. The authors of [16] proposed a model to protect encryption keys based on a concept of distributed keys representation. The first key part stored in the DB, and the other part is obtained by converting the user password. Elovici et al. presented a special DB encryption model that permits to protect keys using "Wallet" mechanism [17], although Itimar et al. used asymmetric key encryption [18]. The authors of [19] suggested a solution called "Server-HSM" which merges an HSM and a security server into a module called "HW Security Module" that might be integrated into a DB server. This module manages user privileges and protects keys with their encryption. El bouchti et al. presented a full package of encryption keys protection models inside the DBMS. Their method allows encrypting keys with master keys generated according to encryption granularity adopted by the DBMS encryption model [15]. Sesay et al. in their proposed 
DB encryption model suggested protecting keys by the use of a particular way. In fact, they generate the encryption keys from a unique master key $(\mathrm{Km})$ generated and stored in a tamper-proof controller [20].

Notwithstanding several DB security studies for improving the concept of encryption keys protection within DBMS, more investigations are required to develop, improve, and provide more security and flexibility to keys protection. Then, as discussed in our previous work presented in $[7,10,15]$, most of the proposed solutions have their advantages and disadvantages. However, to our knowledge, a trusted and simple solution concretized by a real implementation, and adapted to more than one DB encryption model has not yet been proposed.

In this context, the present work aims to implement two solutions proposed in our previous work [15] to protect DB encryption keys. Our solutions protect encryption keys when encryption granularity adopted by the DBMS is fixed at the column level or the entire table level. They consist of encrypting the DB encryption keys of tables and columns using Km, generated by deploying two models. We consider that the main original features of our solution are its reliability and its principle. It does not require any control or management of key protection by a DB or security administrator as well as it resists strongly against attacks performed by administrators.

The present work will be structured as follows: section two presents the proposed models of $\mathrm{Km}$ and the common objects of the implementation of each model. It also explains how our $\mathrm{Km}$ models work with DB encryption models that we will implement. Section three explains the implementation and discusses the provided results. Finally, our article ends with a conclusion.

\section{Definition of the proposed solution}

In this section, we will define two models (1 and 2) of the Km generation that protects, respectively, the columns and tables keys. We will also describe the role of the common objects that have used between the two models' implementation and how those models work in an encryption/decryption process.

\subsection{Proposed models of Km}

The generation of the Km follows the two different models below:

- The Km (C) key used to protect the encryption keys of the DB columns. It is generated by the DBMS according to the model defined below:

$\mathrm{Km}(\mathrm{C})=H($ Table_name $\|$ Column_name $)(1)$

- $\quad$ The $\mathrm{Km}(\mathrm{T})$ key utilized to protect the encryption keys of the DB tables. It is generated by the DBMS according to the model defined below:

$K m(T)=H($ Table_name $\|$ Database_name $)(2)$
In order to concretize the functioning and the response of the models (1) and (2), we have designed and implemented two models of DB encryption (A) and (B) having, respectively, two encryption levels: column and table. The functioning of each $\mathrm{Km}$ generation model is associated with the execution of a DB encryption model, as shown in Table 1.

Table 1: The generation model of $\mathrm{Km}$ and the DB encryption model associated

\begin{tabular}{|l|l|}
\hline \multicolumn{1}{|c|}{ Km generation model } & \multicolumn{1}{c|}{$\begin{array}{c}\text { Associated DB encryption } \\
\text { model }\end{array}$} \\
\hline $\begin{array}{l}\text { Model (1): Km generation } \\
\text { model for protecting } \\
\text { encryption keys of columns }\end{array}$ & $\begin{array}{l}\text { Model (A): DB encryption } \\
\text { model at the column level }\end{array}$ \\
\hline $\begin{array}{l}\text { Model (2): Km generation } \\
\text { model for protecting } \\
\text { encryption keys of tables }\end{array}$ & $\begin{array}{l}\text { Model (B): DB encryption } \\
\text { model at table level }\end{array}$ \\
\hline
\end{tabular}

\subsection{The common objects of each model implementation}

The creation of elements below is common for the implementation of the models (1) and (2).

MYTABLE_ENCRYPT_OBJET: This DB table contains records of : i) the names of the objects on which encryption has defined, ii) the used encrypt algorithms, and iii) the encryption of the encryption keys using $\mathrm{Km}$. It has the following structure:

\section{MYTABLE_ENCRYPT_OBJET (K_OBJECT_NAME, K_ENCRYPT_ALGO, K_KEY)}

TEST_MANAGEMENT: This DB table contains the records of : i) the names of the objects on which encryption has defined, ii) the used encrypt algorithms, iii) the object encryption keys, and iv) the $\mathrm{Km}$ of each object name. In fact, TEST_MANAGEMENT table is not implemented in the real working case of our solution. Nevertheless, its main role is to illustrate the results of the creation of both encryption keys and the $\mathrm{Km}$. The table has the following structure:

\section{TEST_MANAGEMENT (C1_OBJECT, C2_ALGO, C3_KEY, C4_MASTERKEY)}

where:

C1_OBJECT, K_OBJECT_NAME: the object on which we have defined encryption;

C2_ALGO, K_ENCRYPT_ALGO: the algorithm used for encryption;

C3_KEY: the encryption key;

C4_MASTERKEY: the generated Km;

$\mathrm{K} \_$KEY: the encrypting result of encryption keys using $\mathrm{Km}$.

The Md5 hash function: it is used to create the encryption keys and the $\mathrm{Km}$ while implementing all models. 
The AES256 algorithm: it is the algorithm used to encrypt / decrypt the data.

Note: The term "Objet" signifies the column and table names.

\subsection{Principle of the encryption/decryption process}

An encryption/decryption process in the model (A) follows the defined steps below:

When a user sends a query to be executed, the DBMS generates the $\mathrm{Km}$ for each column on which encryption is defined. The DBMS decrypts, using the generated $\mathrm{Km}$, a value from the K_KEY column belonging to MYTABLE_ENCRYPT_OBJET table and which corresponds to the column desired to be encrypted or decrypted. This process generates the real encryption key of column that will encrypt (in the case of an inserting or updating query) or decrypt (in the case of a consulting query) the data.

The encryption/decryption process in the model (B) follows similar operations. In this case, the DBMS generates a single Km in response to the user's query since the encryption is defined on the entire table.

\subsection{Case study: the dosimetric monitoring of agents}

A real case study has chosen to illustrate the models' implementation results.

Let have a database named "ORCL10G" of a nuclear power plant intended to manage the dosimetric monitoring of agents working under ionizing radiation. The "agent" table stores the accumulation of the different types of doses received by each agent during the period of his work within the plant. We assume that all the data in the "agent" table are sensitive since the dose values are considered in the nuclear field as medical secret. The table "agent" has the following structure:

agent (idf_agent, name_agt, Dose_interne_agt,

Dose_superficielle_agt, Dose_profonde_agt, Catégorie_agt)

\section{Implementation of the proposed models}

In this section, we will present the implementation of the models (1) and (2) generating $\mathrm{Km}$ as well as the corresponding DB encryption models (A) and (B).

\subsection{Implementation of the model (1)}

In order to create the "agent" table and defining encryption on all its sensitive columns, we have used the following SQL syntax:
Create table agent (idf_agent varchar2(100) encrypt using AES256, name_agt varchar2(100) encrypt using AES256, Dose_interne_agt varchar2(100) encrypt using AES256, Dose_superficielle agt varchar2(100) encrypt using AES256, Dose_profonde_agt varchar2(100) encrypt using AES256, Catégorie_agt varchar2(100) encrypt using AES256);

The "Algo1" algorithm supports the execution task of this statement; it creates the "agent" table and defines encryption on its columns using the AES256 algorithm. In fact, the data column will be encrypted/decrypted with six keys $\mathrm{K}_{\mathrm{C}}$ which will be protected by six masters key $\mathrm{Km}(\mathrm{C})$. In addition, the "Algo1" generates and stores the encryption keys of the columns $(\mathrm{Kc})$ and the master keys $(\mathrm{Km}$ (C)) within MYTABLE_ENCRYPT_OBJET and TEST_MANAGEMENT according to the models defined below:

/* The model of the encryption key used in the model $(\mathrm{A}) * /$ $\mathrm{Kc}=\mathrm{H}$ (Column_name)

/* The Km generation model of a column */ $\mathrm{Km}(\mathrm{C})=\mathrm{H}$ (Table_name $\|$ Column_name)

Algorithm1: Process managed by Algo1.

\begin{tabular}{|l|}
\hline Algo1 \\
\hline Input: Sensitive_column_query \\
Output:Created_sensitive_column_query \\
Begin \\
Loop \\
Decompose (Sensitive_column_query); \\
Sensitive_column_name := Extract (Sensitive_column \\
query); \\
Kc:= Kc_Generator (Sensitive_column_name); \\
Km (C):=Km_Generator (Sensitive_column_name, Table \\
name); \\
End loop; \\
Insert into TEST_MANAGEMENT values \\
(Sensitive_column_name, Used_algo, Kc, Km (C)); \\
Insert into MYTABLE_ENCRYPT_OBJET values \\
(Sensitive_column_name, Used_algo, Encrypt_AES256 (Km \\
(C), Kc )); \\
Execute (Sensitive_column_query); \\
End;
\end{tabular}

The execution of the "Algo1"algorithm generates the following records:

Table 2: Records created in the TEST_MANAGEMENT table in the model (1).

\begin{tabular}{|c|l|c|c|}
\hline C1_OBJECT & 2_ALGO & C3_KEY & C4_MASTERKEY \\
\hline idf_agent & AES256 & 46D18AC7BD06518B5A33C650CA760D9C & 36EE05BA4ACDEC7D1C07D16FCDCC9CBF \\
\hline name_agt & AES256 & 4BF0A8B1EB8CA12C2912ED25E4D4BDC5 & F8422E273F1957702DE160662923C0EA \\
\hline Dose_interne_agt & AES256 & C8FE8472457B1A9436EE90E6D022178F & 452C55A53935CF7056CE1C293FE8D4FC \\
\hline Dose_superficielle_agt & AES256 & 1FD82F97BE7BA2565D7FDD4BD6A91179 & 44BB0C031437DB46B641257337C7C458 \\
\hline Dose_profonde_agt & AES256 & F67D1D0A822D37E3AC03D69C94A38994 & 8D71448C964377D9F0E539B3FB230133 \\
\hline Catégorie_agt & AES256 & 822E1CDA2CFA7B1EF36B90523E337682 & FE30063A2D96A6DB6059CE708103BD5F \\
\hline
\end{tabular}


K. El bouchti et al. / Advances in Science, Technology and Engineering Systems Journal Vol. 5, No. 2, 87-94 (2020)

Table 3: Records created in the MYTABLE_ENCRYPT_OBJET table in the model (1).

\begin{tabular}{|l|c|c|}
\hline K_OBJECT_NAME & K_ENCRYPT_ALGO & K_KEY \\
\hline idf_agent & AES256 & 12BB4076B53A907324D42AFB9B0501006A93E1F347EC05536A7115E4554 \\
& & CD8D06662D1EEEAC1CA8D71BC2A33EFD7810B \\
\hline name_agt & AES256 & 90F4D05F7C5A11A5752E8AB1354BE82D797BFE16051F78DD7018A60856 \\
& & 49A709515637DB88472F14509DB73FC76E6B0C \\
\hline Dose_interne_agt & AES256 & 6B40D18BF99C712F4F4034E8A11AF73FC8B422CCC218AF595FD06EB4 \\
& & E4AE4BB3E8D5D0CCA9BE57434FCD690A577D05D0 \\
\hline Dose_superficielle_agt & AES256 & 5F03CF21D034BFFBA1FEA4BD5CEA43A15A32E8C9F4FDE591AF842995B \\
& & BD30FF53CC25849363BCF54B8FC2E28FD7FF7A7 \\
\hline Dose_profonde_agt & AES256 & 3A468DD545A12883543199A45202E4CE3AAA4D006CE4453BFC380377423 \\
& & C24C3FA85A1BF68F31F6E011C062C5E19D2AE \\
\hline Catégorie_agt & AES256 & 34FEFD552E0D63C4A99281FC1B0A8189D5B527EDC303E9AB760B35C1A2 \\
& & 28C1F7B0EFDAAF26B7E3F507297D351E64FFE9 \\
\hline
\end{tabular}

Table 4: The "agent" table before encryption.

\begin{tabular}{|l|l|l|l|l|l|}
\hline idf agent & name_agt & Dose_interne_agt & Dose_superficielle_agt & Dose_profonde_agt & Catégorie_agt \\
\hline 1000 & Azzaoui & 10 & 15 & 25 & A \\
\hline 1001 & Rachidi & 8 & 11 & 12 & A \\
\hline 1002 & Kharmoum & 16 & 05 & 14 & A \\
\hline 1003 & Sajid & 14 & 66 & 65 & B \\
\hline
\end{tabular}

Table 5: The "agent" table after encryption.

\begin{tabular}{|c|c|c|c|c|c|}
\hline idf agent & name_agt & Dose_interne_agt & $\begin{array}{l}\text { Dose_superficielle_ } \\
\text { agt }\end{array}$ & Dose_profonde_agt & Catégorie_agt \\
\hline 5754957F70316A9C02 & 745A2F1FF2BDAD322 & B5063DD0A036503D0 & FDBD02C08C389AE7 & D694729051643896225 & D1D88EDED1CF67BF \\
\hline 01002A8CBFE412 & 6910989994A7287 & 2F11B63DF1B12B1 & 583F8211E428B2A7 & 89DA79E580A60 & $3 \mathrm{AB} 34261052 \mathrm{FF} 335$ \\
\hline A6776D65772FBF4992 & 1C65506D1F8AA015B & 5C6ABEA8480996813 & BD706EE5E149EBDC & BECBC7C7B6BD0B0E & D1D88EDED1CF67BF \\
\hline D8AFC2A2B387C9 & 5B98765133E9782 & 19175A05C4B1AB5 & E6796457E944FB81 & 12CF1AD549E22682 & $3 \mathrm{AB} 34261052 \mathrm{FF} 335$ \\
\hline AFBFEA3BC5FCD283 & 67BC01E3BF672D7BF & B8C892229BBB1E395 & 125180FAB503F868E & A8A68F1B4D1D3DD0F & D1D88EDED1CF67BF \\
\hline 96E974016169DF5D & 568ACC3A582ECB8 & 1290107C524B12A & DC55F42D0BB34CB & 398AFED4A54A0A5 & $3 \mathrm{AB} 34261052 \mathrm{FF} 335$ \\
\hline 14DB0A7462832F494E & 7A1FBFCD0E02BC191 & 23BF97F16393EC6A8 & 293A72927BD5C9760 & A2B800E15CAFD3AFA & 5315BF4B7B530AA72 \\
\hline AC7C8D12A09FC6 & 0055CF76279F21A & 4B93FF4F9EB74AA & 450FFFF14CEC693 & 9A22FB0A4C257B8 & 9AC1CDCB53F4C8B \\
\hline
\end{tabular}

In order to test model (1), the "Algo2" algorithm represents the functioning of Model (A). It supports the data encryption inserted by a user. Tables 4 and 5 show the result of inserting four lines in the "agent" table before and after the encryption.

Algorithm 2: The DB encryption using the model (1) and (A).

\begin{tabular}{|l|}
\hline Algo2 \\
\hline CREATE OR REPLACE TRIGGER Insert_Model_A \\
BEFORE INSERT ON agent \\
FOR EACH ROW \\
DECLARE \\
\hline
\end{tabular}

$\underline{\text { www.astesj.com }}$
Kc1 varchar2(100);

$\mathrm{Kc} 2$ varchar2(100);

Kc3 varchar2(100);

Kc4 varchar2(100);

Kc5 varchar2(100);

Kc6 varchar2(100);

BEGIN

$/ *$ Generating $\mathrm{Km}$ and seeking Kc for each column from MYTABLE ENCRYPT OBJET*/ 
Kc1:= Search_Encrypt_Key ('idf_agent');

Kc2:= Search_Encrypt_Key ('name_agt');

Kc3:=Search_Encrypt_Key ('Dose_interne_agt');

Kc4:= Search_Encrypt_Key ('Dose_superficielle_agt');

Kc5:=Search_Encrypt_Key ('Dose_profonde_agt');

Kc6:= Search_Encrypt_Key ('Catégorie_agt');

$$
\text { /* Inserting in table "agent"*/ }
$$

INSERT INTO agent VALUES (Encrypt_AES256

(Kc1, :new.idf_agent), Encrypt_AES256

(Kc2, :new.name agt, Encrypt_AES256

(Kc3, :new.Dose_interne_agt), Encrypt_AES256

(Kc4, :new.Dose_superficielle_agt), Encrypt_AES256

(Kc5, :new.Dose_profonde_agt), Encrypt_AES256

(Kc6, :new.Catégorie_agt));

End ;

\subsection{Implementation of the model (2)}

To implement model (2), we define encryption on the "agent" table level using the following SQL syntax:

Create table agent encrypt using AES256 (idf_agent varchar2(100), name_agt varchar2(100), Dose_interne_agt varchar2(100), Dose_superficielle_agt varchar2(100), Dose_profonde_agt varchar2(100), Catégorie_agt varchar2(100));

The "Algo3" algorithm supports the execution of this instruction. It creates the "agent" table and defines encryption on all its data using the algorithm AES256. In this case, the data are encrypted /decrypted with a single key $\mathrm{K}_{\mathrm{T}}$, which will be protected by a single master key $\mathrm{Km}(\mathrm{T})$. The "Algo3" algorithm generates and stores $\mathrm{K}_{\mathrm{T}}$ and $\mathrm{Km}$ (T) also in MYTABLE_ENCRYPT_OBJET and TEST_MANAGEMENT according to the models defined below:

/* The model of the encryption key used in the model (B)*/ $\mathrm{K}_{\mathrm{T}}=\mathrm{H}$ (Table_name)

/* The Km generation model of a table */ $\mathrm{Km}(\mathrm{T})=\mathrm{H}$ (Table_name $\|$ Database_name)

Algorithm 3: Process managed by Algo3.

\begin{tabular}{|l|}
\hline Algo3 \\
\hline Input: Sensitive_table_query \\
Output: Created_sensitive_table_query \\
Begin
\end{tabular}

Decompose (Sensitive_table_query);

Sensitive_table_name:=Extract (Sensitive_table_query);

$\mathrm{K}_{\mathrm{T}}:=\mathrm{K}_{\mathrm{T}}$ _Generator (Sensitive_table_name);

$\mathrm{Km}(\mathrm{T}):=\mathrm{Km} \_$Generator (Sensitive_table_name, Database name);

Insert into TEST_MANAGEMENT values

(Sensitive table_name, Used_algo, $\mathrm{K}_{\mathrm{T}}, \mathrm{Km}(\mathrm{T})$ ); Insert into MYTABLE_ENCRYPT_OBJET values

(Sensitive_table_name, Used_algo, Encrypt_AES256

$\left.\left(\mathrm{Km}(\mathrm{T}), \overline{\mathrm{K}}_{\mathrm{T}}\right)\right)$;

Execute (Sensitive_table_query);

End ;

The execution of the "Algo3" algorithm generates the following records:

The "Algo4" algorithm represents the functioning of the model (B). It supports the data encryption inserted by a user. The tables 8 and 9 show the result of inserting four rows in the "agent" table before and after the encryption.

Algorithm 4: The DB encryption using model (2) and (B).

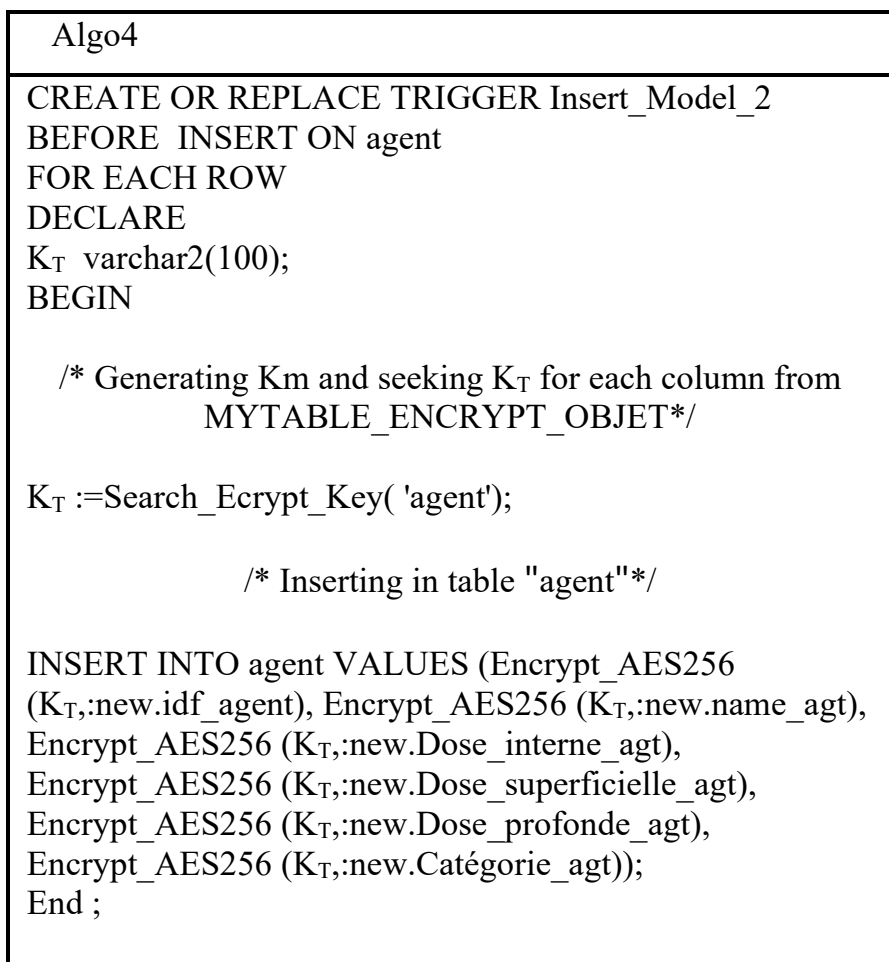

Table 6: Records created in the TEST_MANAGEMENT table in the model (2).

\begin{tabular}{|c|c|c|c|}
\hline C1_OBJECT & C2_ALGO & C3_KEY & C4_MASTERKEY \\
\hline agent & AES256 & B33AED8F3134996703DC39F9A7C95783 & 697C371A913425CF202D15F143D2DAF0 \\
\hline
\end{tabular}

Table 7: Records created in the MYTABLE_ENCRYPT_OBJET table in the model (2).

\begin{tabular}{|c|l|c|}
\hline K_OBJECT_NAME & K_ENCRYPT_ALGO & K_KEY \\
\hline agent & AES256 & 736079082547466884631FC41910AB5770A6962367465EC1CB9526A \\
& & 864367916929D05016D02B96D9B55511854D3AB13 \\
& & \\
\hline
\end{tabular}

www.astesj.com 
K. El bouchti et al. / Advances in Science, Technology and Engineering Systems Journal Vol. 5, No. 2, 87-94 (2020)

Table 8: The "agent" table before encryption.

\begin{tabular}{|l|l|l|l|l|l|}
\hline idf agent & name_agt & Dose_interne_agt & Dose_superficielle_agt & Dose_profonde_agt & Catégorie_agt \\
\hline 1000 & Azzaoui & 25 & 25 & 25 & A \\
\hline 1001 & Rachidi & 8 & 11 & 12 & A \\
\hline 1002 & Kharmoum & 16 & 05 & 14 & A \\
\hline 1003 & Sajid & 14 & 66 & 65 & B \\
\hline
\end{tabular}

Table 9: The "agent" table after encryption.

\begin{tabular}{|c|c|c|c|c|c|}
\hline idf agent & name_agt & Dose_interne_agt & Dose_superficielle_ag & Dose_profonde_agt & Catégorie_agt \\
\hline 3F843D72B4CF6AF3B & 03377847D26334CDA6 & 7012CB55124F226FA2 & 7012CB55124F226FA2 & 7012CB55124F226FA2 & 91B5BD099938E4CE4 \\
FEDD0F8A73A46DB & 65F815335C87F9 & E530E0D8133F14 & E530E0D8133F14 & E530E0D8133F14 \\
DF76529F6740B8A \\
\hline 2D80DCE15D394B765 & 0B71021170A841DAE & 0C04B7B8C2A594ED2 & 52516FBF14B5DB600 & CF92FB85EF0E373795 & 91B5BD099938E4CE4 \\
94AD5E18F3405BE & 0CB4641C08076CD & D6CE2FCD9EE91FD & CF294F47153C168 & F9C4D57D66ECF1 & DF76529F6740B8A \\
\hline 4CDDFEF4428E61343 & DA45B069E89AC7BE4 & 0F8913B9B06C772A68 & 5C9D1E8240D88EAD & CC2CCB3C2994CE129 & 91B5BD099938E4CE4 \\
AD6C823FF1860A4 & C2B69EE232EB1A4 & 8E9489B13A1124 & 7072C8B7673AB4C1 & 59B9C9B66F478A5 & DF76529F6740B8A \\
\hline CA80281E0ADBD105C & EE6099C500334279227 & CC2CCB3C2994CE129 & 9B07E13AC87C52956 & EC2F5721D81D6CACE & 72621DFBF38C674D9 \\
E371803DD3F575E & AFA5C27E199FB & 59B9C9B66F478A5 & 2187B81CFCD6B2B & 4C6EA7B9B49ED52 & BA24509BDA41160 \\
\hline
\end{tabular}

\subsection{Results and discussion}

This section introduces data discussion and analysis based on the findings obtained by implementing the two Km models. They are summarized as follow:

- The proposed solutions are more practical than the conventional ones, primarily the Wallet, HSM, and the security server, where their disadvantages have explained and revealed in [15]. Our solutions optimize perfectly additional costs to protect the keys either in terms of hardware acquisition (case of HSM and security server) or in human resources (the administrator of the security server).

- The Wallet solution used in Oracle TDE generates Km, which protects encryption keys, and stores it within the Wallet. Here, it is necessary to create the Wallet and its password as well as a secured location (such as backup systems) to store the password whenever the $\mathrm{Km}$ is newly created. This operation is mandatory before starting the process of data encryption/decryption inside DBMS [11]. It is worthy to mention that the backup system is a critic component of the Wallet concept. In this vein, the protection concept of encryption keys based on the proposed models (1) and (2) is similar to the Wallet solution in terms of $\mathrm{Km}$ generation within the DBMS. However, with our concept, the $\mathrm{Km}$ creation does not require any Wallet creation to store $\mathrm{Km}$ or secured location to store the password.

- The proposed solution does not require any protection management of the encryption keys by a security administrator, DB administrator or another trusted collaborator, as discussed in [15]. Hence, none of them knew about the generation of $\mathrm{Km}$ or its location. The probability of attacking keys is almost impossible, even if the attacker arrives to consult table
MYTABLE_ENCRYPT_OBJET stored in the DB dictionary.

- The proposed solution does not define the place where storing $\mathrm{Km}$. $\mathrm{Km}$ generation is performed automatically while defining encryption on a sensitive object (column or table), precisely during the creation by the DB administrator. Obtaining a value of $\mathrm{Km}$ by attackers (administrators, internal or external attackers) is almost an impossible operation.

- It is important to notice that the new concept we have proposed and implemented enhances the security of encryption keys. In fact, compared to the Oracle TDE Column Encryption solution that uses a single master key to protect all the column keys, the model (1) generates several $\mathrm{Km}$ to protect each column key. The number of $\mathrm{Km}$ generated is equal to the number of sensitive columns. For example, if a DB contains 20 sensitive columns to encrypt, we need 20 keys to encrypt data and $20 \mathrm{Km}$ to protect them.

- Our solution can work with any DB encryption models, obviously with those implementing encryption granularities at the level of columns and tables. It is well adapted to free license DBMSs.

- In the model (1) implementation, we focused on to protect 6 encryption keys of the following columns (idf_agent, name_agt,Dose_interne_agt,Dose_superficielle_agt,Dose profonde_agt, Catégorie_agt). Each column key is protected by encrypting it with its own $\mathrm{Km}$ generated by the model (1). This protection was tested by the implementation of the model (A). Table 4 shows the encryption test results of the 6 columns of the "agent" table deploying the model (1). Likewise, in the model (2) implementation, one encryption key of the table "agent" has protected with a single $\mathrm{Km}$ generated using this model. Then, this protection was tested using the model (B). Table 
8 represents the result obtained of encrypting table "agent" by deploying the model (2).

- The results presented in tables 2 and 3 shows the keys generation when the "agent" table is created by the administrator. In table 2, the columns C3_KEY and C4_MASTERKEY represents, respectively, the encryption key generated of each column and its associated $\mathrm{Km}$. In table 3, the column K_KEY, belongs to MYTABLE_ENCRYPT_OBJET table, represents the encryption of each column key of the table "agent" using its associate $\mathrm{Km}$.

- In table 6, the columns C3_KEY and C4_MASTERKEY represents, respectively, the key generated to encrypt the entire table and its $\mathrm{Km}$. The value of the column $\mathrm{K} \_\mathrm{KEY}$ in table 7 represents the encryption of the encryption key using its associate $\mathrm{Km}$. Both tables show the keys generation when the "agent" table is created by the administrator.

- As described earlier, tables 4, 5, 8, and 9 show the result of inserting rows in the "agent" table before and after the encryption. The encryption results showed that our solution works perfectly, either when encrypting or decrypting data. Actually, the encryption process via model (A) or (B) requires the generation of $\mathrm{Km}$ through model (1) or (2), respectively. Each value of generated $\mathrm{Km}$ is used to extract, from K_KEY column, the real encryption /decryption key. The process of data decryption follows the same operations.

- Finally, both proposed model works inside DBMS are summarized according to the algorithm flowchart described below:

Let's consider a sensitive table A

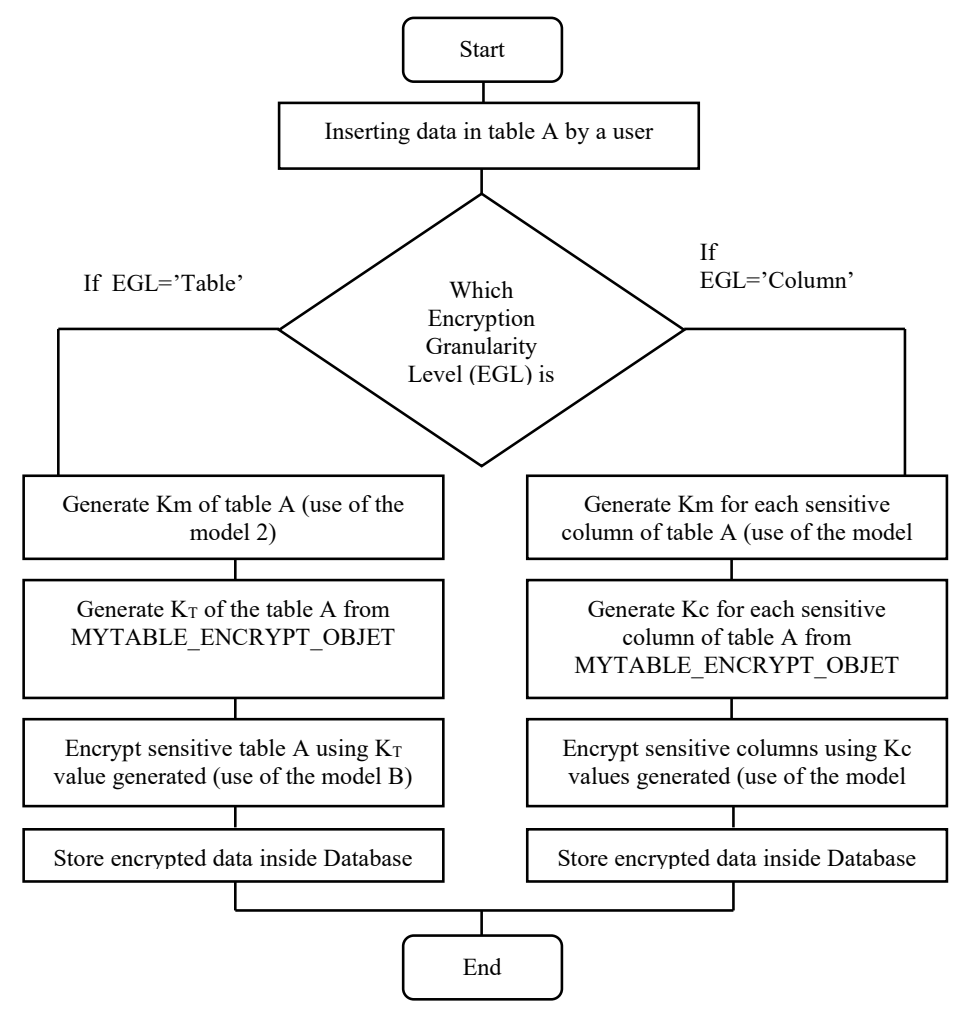

Figure 1: Algorithm flowchart specifying the both proposed models.

\section{Conclusion:}

Besides the conventional mechanisms deployed to secure sensitive DB (network protection, authentification, and access control), data encryption at DBMS level is a strong way that reinforces the defense in depth of the sensitive data. This process is strongly linked to the protection of the encryption keys on which they depend on two main factors: the location where the keys are stored and users who have access to them.

Our contribution in this article is to implement two solutions that secure encryption keys within the DBMS. These solutions are original and well adapted to any encryption model inside a DBMS. The solution's purpose is to protect DB keys by their encryption using a master key generated when defining encryption on a table or column. In forthcoming works, we aim to develop a solution that covers the protection of encryption keys when encryption is done on Tablespaces.

\section{Conflict of Interest}

This manuscript has not been published and is not under consideration for publication elsewhere. We have no conflicts of interest to disclose.

\section{Acknowledgment}

I would like to express my appreciation to all my professors, whom they helped and guided me to realize this work.

\section{References}

[1] E. Shmueli, R. Vaisenberg, Y. Elovici, C. Glezer, "Database encryption: an overview of contemporary challenges and design considerations" ACM SIGMOD Record, New York, NY, USA, 2010. DOI: $10.1145 / 1815933.1815940$

[2] Hashim, Hassan B, "Challenges and Security Vulnerabilities to Impact on Database Systems" Al-Mustansiriyah Journal of Science 29(2), 117-125,2018. DOI: http://doi.org/10.23851/mjs.v29i2.332

[3] S. Jacob, "Protection cryptographique des bases de données: conception et cryptanalyse,“ Ph.DThesis, Université Pierre et Marie Curie-Paris VI, 2005.

[4] A.M.Mostafa, F.A. Almutairi, M.M. Hassan, "False alarm reduction scheme for database intrusion detection system" Journal of Theoretical \& Applied Information Technology., 96(10), 2816-2825. ISSN: 1992-8645

[5] I. Homoliak, J.Guarnizo, Y.Elovici, M.Ochoa, "Insight into insiders and it: A survey of insider threat taxonomies, analysis, modeling, and countermeasures" ACM Computing Surveys, New York, NY, USA 2019. https://doi.org/0000001.0000001

[6] Deepicata. N. Soni, "Database Security: Threats and Security Techniques" International Journal of Advanced Research in Computer Science and Software Engineering., 5(5), 621-624, 2015. ISSN: 2277 128X

[7] K. El bouchti, S.Ziti, Y.Ghazali, N.Kharmoum, "Sécurité des Bases de Données : Menaces principales et solution de chiffrement existantes", in Proceedings of the JDSIRT Conference Information Systems, Networks Telecommunications, Meckness, Morocco, 2018.

[8] K. El bouchti, N. Kharmoum, S. Ziti, F. Omary, "A new approach to prevent internal attacks on Database encryption keys" Proceedings of the International Conference Scientific Days Applied Sciences, Larache, Morocco, 2019

[9] E. Shmueli, R. Vaisenberg, E. Gudes, Y. Elovici, "Implementing a database encryption solution, design and implementation issues" Computers \& security, 44, 33-50, 2014. DOI: ORG/10.1016/J.COSE.2014.03.011

[10] K. El Bouchti, S. Ziti, F. Omary, N. Kharmoum, "A New Database Encryption Model Based on Encryption Classes" Journal of Computer Science., 15(6), 844.854, 2019. DOI: 10.3844 jicssp.2019.844.854

[11] Oracle (2016), Oracle $®$ Database Advanced Security Administrator's Guide 11g Release 2 (11.2)[online] Technical Document:

[12] S. Mukherjee, "Popular SQL Server Database Encryption Choices" International Journal of Computer Science and Engineering, arXiv preprint arXiv: 1901.03179, 2018. ISSN: $2231-2803$

[13] MySQL Server Documentation. MySQL 5.7 Reference Manual Online

[14] A. K. Maurya , A.Singh, U.Dubey, S.Pandey, U. N.Tripathi, "Protection of Data Stored in Transparent Database System using Encryption" Journal of 
Computer and Mathematical Sciences., 10(1), 190-196, 2019. ISSN 23198133.

[15] K. El bouchti, S.ZITI, F.OMARY, "A new approach to protect encryption keys in Database Management System", Proceedings of the International Conference Modern Intelligent Systems Concepts, Rabat, Morocco, 2018.

[16] V.V.Galushka, A.R.Aydinyan, O.L.Tsvetkova, V.A.Fathi, D.V.Fathi, "System of end-to-end symmetric database encryption" In International Conference Information Technologies in Business and Industry, 2018. Doi :10.1088/1742-6596/1015/4/042003.

[17] Elovici, Y., Vaisenberg, R., \& Shmueli, E. (2018). U.S. Patent No. 9,934,388. Washington, DC: U.S. Patent and Trademark Office.

[18] Itamar, E., \& Rotem, A. (2018). U.S. Patent Application No. 15/570,775.

[19] L.Bouganim, Y.Guo, Database encryption. In Encyclopedia of Cryptography and Security, Springer US, 2011

[20] S.Sesay, Z. Yang, J.Chen, D. Xu, "A secure database encryption scheme" In Consumer Communications and Networking Conference, CCNC. 2005 Second IEEE, Las Vegas, NV, USA, 2015. DOI: $10.1109 /$ CCNC.2005.1405142 